\title{
Spectral Gap of the Largest Eigenvalue of the Normalized Graph Laplacian
}

\author{
Jürgen Jost ${ }^{1} \cdot$ Raffaella Mulas $^{1}$ (D) Florentin Münch $^{1}$
}

Received: 9 January 2020 / Revised: 29 March 2020 / Accepted: 23 May 2020 / Published online: 5 April 2021

(c) The Author(s) 2021

\section{Abstract}

We offer a new method for proving that the maxima eigenvalue of the normalized graph Laplacian of a graph with $n$ vertices is at least $\frac{n+1}{n-1}$ provided the graph is not complete and that equality is attained if and only if the complement graph is a single edge or a complete bipartite graph with both parts of size $\frac{n-1}{2}$. With the same method, we also prove a new lower bound to the largest eigenvalue in terms of the minimum vertex degree, provided this is at most $\frac{n-1}{2}$.

Keywords Spectral graph theory · Normalized Laplacian · Largest eigenvalue · Sharp bounds

Mathematics Subject Classification $05 \mathrm{C} 50 \cdot 15 \mathrm{~A} 42$

\section{Introduction}

Spectral graph theory investigates the fundamental relationships between geometric properties of a graph and the eigenvalues of the corresponding linear operator. A general overview is given in [2]. In terms of the largest eigenvalue, the normalized graph Laplacian is particularly interesting as it measures how close a graph is to a bipartite graph. In this paper, we are interested in the reverse question, i.e., how far from a bipartite graph a graph can be. This translates to giving lower bounds on the largest eigenvalue. Several bounds are given in [4]. However, the best known estimate

Raffaella Mulas

Raffaella.Mulas@mis.mpg.de

Jürgen Jost

jost@mis.mpg.de

Florentin Münch

muench@mis.mpg.de

1 Max Planck Institute for Mathematics in the Sciences, Leipzig, Germany 
so far is $\lambda_{n} \geq \frac{n}{n-1}$ which is only attained if the graph is a complete graph [2]. Here, $\lambda_{n}$ denotes the largest eigenvalue and $n$ is the number of vertices.

Naturally, the question arises what is the optimal a priori estimate for $\lambda_{n}$ for noncomplete graphs. Das and Sun [3] proved that for all non-complete graphs one has

$$
\lambda_{n} \geq \frac{n+1}{n-1},
$$

with equality if and only if the complement graph is a single edge or a complete bipartite graph with both parts of size $\frac{n-1}{2}$. Here, we offer a new method for proving these results, see the proofs of Theorems 2.1 and 3.1. Furthermore, we use this new method for showing that for a graph with minimum vertex degree $d_{\min } \leq \frac{n-1}{2}$,

$$
\lambda_{n} \geq 1+\frac{1}{\sqrt{d_{\min }\left(n-1-d_{\min }\right)}} .
$$

\section{Eigenvalue Estimate}

A graph $G=(V, E)$ consists of a finite non-empty vertex set $V$ and a symmetric edge relation $E \subset V \times V$ containing no diagonal elements $(v, v)$. We write $v \sim w$ for $(v, w) \in E$. Let $G=(V, E)$ be a graph with $n$ vertices. The vertex degree is denoted by $d(v):=|N(v)|$ where $N(v):=\{w \in V: w \sim v\}$. We also denote $N_{k}(v):=\{w \in V: d(v, w)=k\}$ where $d(v, w):=\inf \left\{n: v=x_{0} \sim \cdots \sim x_{n}=w\right\}$ is the combinatorial graph distance. We say $G$ is connected if $d(v, w)<\infty$ for all $v, w \in V$. We write $C(V)=\mathbb{R}^{V}$ and we denote the positive semidefinite normalized Laplacian by $L: C(V) \rightarrow C(V)$; it is given by

$$
L f(x):=\frac{1}{d(x)} \sum_{y \sim x}(f(x)-f(y)) .
$$

We will always assume that $d(x) \geq 1$ for all $x \in V$ as the Laplacian is not well defined otherwise. The inner product is given by

$$
\langle f, g\rangle:=\sum_{x} f(x) g(x) d(x)
$$

The operator $L$ is self-adjoint w.r.t. this inner product, and the eigenvalues of $L$ are

$$
0=\lambda_{1} \leq \lambda_{2} \leq \cdots \leq \lambda_{n}
$$

In this paper, we are interested in estimating the largest eigenvalue $\lambda_{n}$ which, by the min-max principle, can be written as

$$
\lambda_{n}=\sup _{f \in C(V) \backslash\{0\}} \frac{\langle L f, f\rangle}{\langle f, f\rangle} .
$$


It is well known that

$$
\frac{n}{n-1} \leq \lambda_{n} \leq 2
$$

where the first inequality is an equality only for the complete graph, and the latter inequality is an equality only for bipartite graphs. The following theorem was established in [3] and gives the optimal a priori lower bound on $\lambda_{n}$ for all non-complete graphs. In contrast to [3, Theorem 3.1], our proof methods are completely different and allow for an extension of this estimate in terms of the minimal vertex degree, see Sect. 4.

Theorem 2.1 [3, Theorem 3.1] Let $G=(V, E)$ be a non-complete graph with $n$ vertices.

Then,

$$
\lambda_{n} \geq \frac{n+1}{n-1}
$$

Proof We first assume that $G$ is connected. Since $G$ is not complete, there exists a vertex $v$ with $d(v) \leq n-2$. As the graph is connected, we have $N_{2}(v) \neq \emptyset$. Let $w \in N_{2}(v)$. Then, $d(w) \leq n-2$ as $v$ and $w$ are not adjacent. Moreover, $N(v) \cap N(w) \neq \emptyset$.

We write $A:=|N(v) \cap N(w)|$. We aim to find a function $f$ with $\langle f, L f\rangle \geq$ $\frac{n+1}{n-1}\langle f, f\rangle$. To do so, it is convenient to choose $f$ in such a way that $L f=\frac{n+1}{n-1} f$ in $v$ and $w$. Particularly, let $f: V \rightarrow \mathbb{R}$ be given by

$$
f(x):= \begin{cases}-1 & : x \in N(v) \cap N(w) \\ \frac{n-1}{2} \frac{A}{d(v)} & : x=v \\ \frac{n-1}{2} \frac{A}{d(w)} & : x=w \\ 0 & : \text { otherwise. }\end{cases}
$$

We observe

$$
d(v) L f(v)=d(v) f(v)+A=\frac{n+1}{2} A
$$

and thus, $L f(v)=\frac{n+1}{n-1} f(v)$. Similarly, $L f(w)=\frac{n+1}{n-1} f(w)$. We now claim that $-L f(x) \geq \frac{n+1}{n-1}$ for all $x \in N(v) \cap N(w)$. We observe $A \geq 1 \vee(d(v)+d(w)+2-n)$ where $\vee$ denotes the maximum, and we calculate

$$
\begin{aligned}
-L f(x) & =\frac{d(x)-|N(x) \cap N(v) \cap N(w)|+f(v)+f(w)}{d(x)} \\
& \geq 1+\frac{1-A+f(v)+f(w)}{d(x)} .
\end{aligned}
$$


As $f(v)+f(w) \geq A$, we can use $d(x) \leq n-1$ and continue

$$
\begin{aligned}
\frac{1-A+f(v)+f(w)}{d(x)} & \geq \frac{1-A}{n-1}+\frac{A}{2 d(v)}+\frac{A}{2 d(w)} \\
& =\frac{1}{n-1}+A\left(\frac{1}{2 d(v)}+\frac{1}{2 d(w)}-\frac{1}{n-1}\right) .
\end{aligned}
$$

Since $d(v) \leq n-2$ and $d(w) \leq n-2$, we see that the term in brackets is positive, and thus,

$$
\begin{aligned}
& A\left(\frac{1}{2 d(v)}+\frac{1}{2 d(w)}-\frac{1}{n-1}\right) \\
& \quad \geq[1 \vee(d(v)+d(w)+2-n)]\left(\frac{1}{2 d(v)}+\frac{1}{2 d(w)}-\frac{1}{n-1}\right) .
\end{aligned}
$$

We write $D:=(d(v)+d(w)) / 2$, and by the harmonic-arithmetic mean estimate, we have $\frac{1}{2 d(v)}+\frac{1}{2 d(w)} \geq \frac{1}{D}$ and thus,

$$
A\left(\frac{1}{2 d(v)}+\frac{1}{2 d(w)}-\frac{1}{n-1}\right) \geq[1 \vee(2 D+2-n)]\left(\frac{1}{D}-\frac{1}{n-1}\right)
$$

We aim to show that the latter term is at least $\frac{1}{n-1}$ which, by multiplying with $D(n-1)$ and subtracting $D$, is equivalent to

$$
[1 \vee(2 D+2-n)](n-1-D)-D \geq 0
$$

If $D \leq \frac{n-1}{2}$, then the maximum equals 1 and the inequality follows immediately. If $D \geq \frac{n-1}{2}$, then we can discard the " $1 \vee$ ", and so the left-hand side becomes a concave quadratic polynomial in $D$ with its zero points in $D=n-2$ and $D=\frac{n-1}{2}$. Thus, the inequality (2.5) holds true for all $D$ between the zero points. Moreover by assumption, $D$ has to be between the zero points which proves the claim that $-L f(x) \geq \frac{n+1}{n-1}$ for all $x \in N(v) \cap N(w)$. Particularly, this shows that $f L f \geq \frac{n+1}{n-1} f^{2}$. Integrating proves the claim of the theorem for all connected graphs.

For non-connected graphs, the smallest connected component has at most $\frac{n}{2}$ vertices. By the standard estimate $\lambda_{n} \geq \frac{n}{n-1}$ applied to the smallest connected component, and the fact that the right-hand side of the estimate is a decreasing function of $n$, we get

$$
\lambda_{n} \geq \frac{n / 2}{n / 2-1}=\frac{n}{n-2}>\frac{n+1}{n-1}
$$

which proves the theorem for non-connected graphs.

Remark 2.2 Das and Sun's proof method of Theorem 2.1 follows two main steps: 


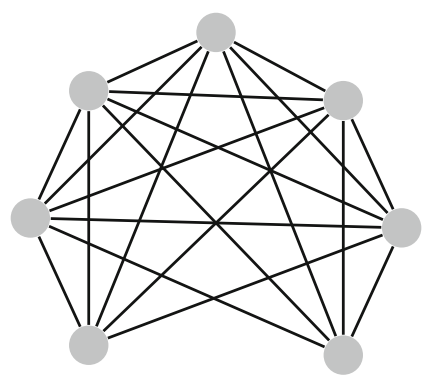

(a)

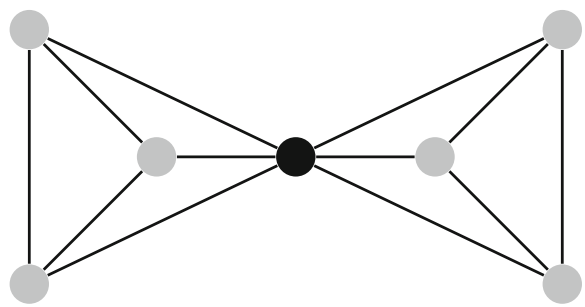

(b)

Fig. 1 For $n=7$, these are the two graphs in Theorem 3.1. The graph on the left is the complete graph $K_{7}$ with one edge removed. The graph on the right is made by two copies of the complete graph $K_{3}$, joined by the black vertex in the middle

1. They consider the cases when the path $P_{4}$, the cycle $C_{4}$ and the star graph $S_{4}$ are induced subgraphs of $G$. For each of these three cases, they find appropriate functions on the vertex set showing that $\lambda_{n}>\frac{n+1}{n-1}$.

2. They use a characterization of $\left\{P_{4}, C_{4}, S_{4}\right\}$-free graphs given in [5], and they study the entire spectrum of such graphs, in order to prove the claim.

The last step, in particular, also includes the characterization of the extremal graphs that we shall prove in Theorem 3.1.

\section{Rigidity}

We now prove that Theorem 2.1 gives the optimal bound, and we characterize equality in the eigenvalue estimate which can be attained only for two different graphs (Fig. 1). One of the graphs is the complete graph with only one edge removed. The other graph is surprisingly significantly different. It can be seen as two copies of a complete graph which are joined by a single vertex. Again, our proof methods differ widely from [3].

Theorem 3.1 [3, Theorem 3.1] Let $G=(V, E)$ be a graph with n vertices. T.f.a.e.:

(i) $\lambda_{n}=\frac{n+1}{n-1}$,

(ii) The complement graph of $G$, after removing isolated vertices, is a single edge or a complete bipartite graph with both parts of size $\frac{n-1}{2}$.

Proof We first prove (i) $\Rightarrow$ (ii). We first note that $G$ is non-complete but connected by the proof of Theorem 2.1. Thus, all inequalities in the proof of Theorem 2.1 must be equalities. Let $v \nsim w$ with $N(v) \cap N(w) \neq \varnothing$. By equality in (2.1), all vertices within $N(v) \cap N(w)$ must be adjacent. By equality in (2.2), all vertices of $N(v) \cap N(w)$ must have degree $n-1$. By equality in (2.3), we obtain

$$
|N(v) \cap N(w)|=(d(v)+d(w)+2-n) \vee 1
$$


By equality in (2.4), we obtain $d(v)=d(w)=D$. Finally, by equality in (2.5), we see that

$$
D \in\left\{\frac{n-1}{2}, n-2\right\} \text {. }
$$

We first show that if $D=n-2$, then the complement graph is a single edge. If $d(v)=d(w)=D=n-2$, then we get $|N(v) \cap N(w)|=n-2$. Since all vertices within $N(v) \cap N(w)$ are adjacent, we see that the only missing edge is the one from $v$ to $w$ which shows that the complement graph is a single edge.

Now we assume $D=\frac{n-1}{2}$. Then, $A=|N(v) \cap N(w)|=1$, and we can write $N(v) \cap N(w)=\{x\}$. We recall that $d(x)=n-1$. We now specify the parts of the bipartite graph which we want to be the complement graph. One part is

$$
P_{v}:=\{v\} \cup N(v) \backslash\{x\}
$$

and similarly, $P_{w}:=\{w\} \cup N(w) \backslash\{x\}$. Let $\widetilde{v} \in P_{v}$ and $\widetilde{w} \in P_{w}$. Then $d(v, \widetilde{w})=$ $d(w, \widetilde{v})=2$ as $x$ is adjacent to every other vertex. By applying the above arguments to the pair $(v, \widetilde{w})$, we see that $N(v) \cap N(\widetilde{w})=\{x\}$. Particularly, $\widetilde{v} \nsim \widetilde{w}$. Moreover, we have $d(\widetilde{w})=d(v)=\frac{n-1}{2}$ and similarly, $d(\widetilde{v})=\frac{n-1}{2}$. By a counting argument, this shows that every $\widetilde{v} \in P_{v}$ is adjacent to every vertex not belonging to $P_{w}$. An analogous statements holds for all $\widetilde{w} \in P_{w}$. Putting everything together, we see that the complement graph of $G$ is precisely the complete bipartite graph with the parts $P_{v}$ and $P_{w}$. This finishes the case distinction, and thus, the proof of the implication (i) $\Rightarrow$ (ii) is complete.

We finally prove (ii) $\Rightarrow$ (i). We start with the case that the complement graph is the complete bipartite graph. Let the parts be $P$ and $Q$. Then, $\phi:=1_{P}-1_{Q}$ is eigenfunction to the eigenvalue $\frac{2}{n-1}$ and every function orthogonal to $\phi$ and 1 is eigenfunction to the eigenvalue $\frac{n+1}{n-1}$.

We end with the case that the complement graph is a single edge $(v, w)$. Then, $\phi=1_{v}-1_{w}$ is eigenfunction to eigenvalue 1 , and $\psi=-2+(n+1)\left(1_{v}+1_{w}\right)$ is eigenfunction to eigenvalue $\frac{n+1}{n-1}$. Every function orthogonal to $\phi, \psi$ and 1 is eigenfunction to the eigenvalue $\frac{n}{n+1}$.

This finishes the proof of (ii) $\Rightarrow$ (i), and thus, the proof of the theorem is complete.

Remark 3.2 In the second equality case in Theorem 3.1, for $n>3$, the eigenvalue $\lambda_{n}$ has multiplicity larger than 1 . With the notation of the proof of that theorem, we can take any vertex $v^{\prime} \in P_{v}$ and any vertex $w^{\prime} \in P_{w}$ and a function that is 1 at $v^{\prime}$ and $w^{\prime}$, -1 at their single joint neighbor $z$, and 0 everywhere else. For $n=5$, that is when we have two triangles sharing a single vertex $z$. We can also take a function that is 0 at $z$ and assume the values \pm 1 on the two other vertices of each of the two triangles, to produce other eigenfunctions with eigenvalue $\frac{3}{2}$.

Remark 3.3 It is known that while the eigenvalues of the non-normalized graph Laplacian decrease or stay the same when an edge is removed, the same does not hold for the normalized Laplacian [1, Remark 9]. The eigenvalues of $L$ may in fact increase 
when an edge is removed, and in this case [1, Theorem 8] gives an upper bound to the increase. This is easy to see, for instance, by the fact that $\lambda_{n} \geq \frac{n}{n-1}$ for every graph, with equality if and only if the graph is complete. Looking at this inequality, however, one may wonder whether the opposite is true for $\lambda_{n}$, i.e., whether the largest eigenvalue of $L$ increases or stays the same when an edge is removed. The answer is no: Theorems 2.1 and 3.1 offer an example that proves this. In particular, let $G$ be a graph given by two copies of a complete graph which are joined by a single vertex. By Theorem 3.1, its largest eigenvalue is $\frac{n+1}{n-1}$. By Theorem 2.1 and Theorem 3.1, any graph given by $G$ with the addition of one edge has largest eigenvalue strictly greater than $\frac{n+1}{n-1}$. Therefore, $\lambda_{n}$ may as well decrease when an edge is removed.

\section{Lower Bound Using the Minimum Degree}

We now use the same method as that in the proof of Theorem 2.1 in order to give a new lower bound to the largest eigenvalue in terms of the minimum vertex degree, provided this is at most $\frac{n-1}{2}$. To the best of our knowledge, this is the first known lower bound to $\lambda_{n}$ in terms of the minimum degree. Li, Guo and Shiu [4] proved a bound in terms of the maximum degree. Namely, they have shown that for a graph with $n$ vertices and $m$ edges

$$
\lambda_{n} \geq \frac{2 m}{2 m-\Delta},
$$

where $\Delta$ is the maximum vertex degree.

Theorem 4.1 Let $G=(V, E)$ be a graph with $n$ vertices and let $d_{\min }$ be the minimum vertex degree of $G$. If $d_{\min } \leq \frac{n-1}{2}$, then

$$
\lambda_{n} \geq 1+\frac{1}{\sqrt{d_{\min }\left(n-1-d_{\min }\right)}} .
$$

Proof Let

$$
\psi:=\psi\left(n, d_{\min }\right):=1+\frac{1}{\sqrt{d_{\min }\left(n-1-d_{\min }\right)}} \text { and } \eta:=\frac{1}{\psi-1} .
$$

We proceed similarly to the proof of Theorem 2.1. We first assume that $G$ is connected. Let $v$ be a vertex of minimum degree, i.e., such that $d(v)=d_{\min }$. As the graph is connected, we have $N_{2}(v) \neq \emptyset$. Let $w \in N_{2}(v)$. Then, $d(w) \leq n-2$ as $v$ and $w$ are not adjacent. Moreover, $N(v) \cap N(w) \neq \emptyset$.

We write $A:=|N(v) \cap N(w)|$. We aim to find a function $f$ with $\langle f, L f\rangle \geq$ $\psi\langle f, f\rangle$. To do so, it is convenient to choose $f$ in such a way that $L f=\psi f$ in $v$ and 
$w$. Particularly, let $f: V \rightarrow \mathbb{R}$ be given by

$$
f(x):= \begin{cases}-1 & : x \in N(v) \cap N(w) \\ \eta \cdot \frac{A}{d(v)} & : x=v \\ \eta \cdot \frac{A}{d(w)} & : x=w \\ 0 & : \text { otherwise. }\end{cases}
$$

We observe

$$
L f(v)=(\eta+1) \cdot \frac{A}{d(v)}=\frac{\eta+1}{\eta} \cdot f(v)=\psi f(v)
$$

and similarly, $L f(w)=\psi f(w)$. We now claim that $-L f(x) \geq \psi$ for all $x \in$ $N(v) \cap N(w)$. We observe $A \geq 1 \vee(d(v)+d(w)+2-n)$ and we calculate

$$
\begin{aligned}
-L f(x) & =\frac{d(x)-|N(x) \cap N(v) \cap N(w)|+f(v)+f(w)}{d(x)} \\
& \geq 1+\frac{1-A+f(v)+f(w)}{d(x)} .
\end{aligned}
$$

In order to proceed, we will use the following lemma which will be proven later independently.

\section{Lemma 4.2 We have}

$$
\frac{\eta}{n-1} \cdot[1 \vee(d(v)+d(w)+2-n)]\left(\frac{1}{d(v)}+\frac{1}{d(w)}-\frac{1}{\eta}\right) \geq \frac{1}{\eta}-\frac{1}{n-1} .
$$

Applying the lemma and using $A \geq 1 \vee(d(v)+d(w)+2-n)$ give

$$
\begin{aligned}
0<\frac{1}{\eta}-\frac{1}{n-1} & \leq \frac{\eta}{n-1} \cdot[1 \vee(d(v)+d(w)+2-n)]\left(\frac{1}{d(v)}+\frac{1}{d(w)}-\frac{1}{\eta}\right) \\
& \leq \frac{\eta A}{n-1} \cdot\left(\frac{1}{d(v)}+\frac{1}{d(w)}-\frac{1}{\eta}\right)
\end{aligned}
$$

Moving $\frac{1}{n-1}$ to the right-hand side and using $d(x) \leq n-1$ give

$$
\begin{aligned}
0<\frac{1}{\eta} & \leq \frac{1}{n-1}+\frac{\eta A}{n-1} \cdot\left(\frac{1}{d(v)}+\frac{1}{d(w)}-\frac{1}{\eta}\right) \\
& \leq \frac{1}{d(x)}+\frac{\eta A}{d(x)} \cdot\left(\frac{1}{d(v)}+\frac{1}{d(w)}-\frac{1}{\eta}\right) \\
& =\frac{1-A+f(v)+f(w)}{d(x)} \\
& \leq-L f(x)-1
\end{aligned}
$$


where we used (4.1) in the last estimate. Thus, $-L f(x) \geq 1+\frac{1}{\eta}=\psi$ for all $x \in$ $N(v) \cap N(w)$. Integrating gives $\langle L f, f\rangle \geq \psi\langle f, f\rangle$ which proves the theorem for all connected graphs. For non-connected graph, we apply the theorem for the connected component containing $v$ and use that $\psi$ is decreasing in $n$. The proof of the theorem is now complete up to the proof of the lemma.

Proof of the Lemma We consider two cases.

1. Case $1 d(v)+d(w) \leq n-1$. Then,

$$
1 \vee(d(v)+d(w)+2-n)=1
$$

and

$$
\frac{1}{d(w)} \geq \frac{1}{n-1-d(v)}
$$

Therefore,

$$
\frac{\eta}{n-1} \cdot\left(\frac{1}{d(v)}+\frac{1}{d(w)}-\frac{1}{\eta}\right) \geq \frac{\eta}{n-1} \cdot\left(\frac{1}{d(v)}+\frac{1}{n-1-d(v)}-\frac{1}{\eta}\right) .
$$

Now, we have that

$$
\begin{aligned}
& \frac{\eta}{n-1} \cdot\left(\frac{1}{d(v)}+\frac{1}{n-1-d(v)}-\frac{1}{\eta}\right) \geq \frac{1}{\eta}-\frac{1}{n-1} \\
& \Longleftrightarrow \\
& \frac{\eta}{n-1} \cdot\left(\frac{1}{d(v)}+\frac{1}{n-1-d(v)}\right) \geq \frac{1}{\eta} \\
& \Longleftrightarrow \\
& \frac{1}{n-1} \cdot\left(\frac{1}{d(v)}+\frac{1}{n-1-d(v)}\right) \geq \frac{1}{\eta^{2}} .
\end{aligned}
$$

This is true by definition of $\eta$ and it is actually an equality.

2. Case $2 d(v)+d(w)>n-1$. Then,

$$
1 \vee(d(v)+d(w)+2-n)=d(v)+d(w)+2-n \geq 2
$$

Therefore, it suffices to prove that

$$
\frac{2 \eta}{n-1} \cdot\left(\frac{1}{d(v)}+\frac{1}{d(w)}-\frac{1}{\eta}\right) \geq \frac{1}{\eta}-\frac{1}{n-1},
$$

i.e.,

$$
\frac{2 \eta}{n-1} \cdot\left(\frac{1}{d(v)}+\frac{1}{d(w)}\right) \geq \frac{1}{\eta}+\frac{1}{n-1},
$$


which can be re-written as

$$
\frac{2}{n-1} \cdot\left(\frac{1}{d(v)}+\frac{1}{d(w)}\right)-\frac{1}{\eta^{2}} \geq \frac{1}{(n-1) \eta} .
$$

In order to prove it, we use the fact that

$$
\frac{1}{\eta^{2}}=\frac{1}{n-1} \cdot\left(\frac{1}{d(v)}+\frac{1}{n-1-d(v)}\right) .
$$

This implies that

$$
\begin{aligned}
& \frac{2}{n-1} \cdot\left(\frac{1}{d(v)}+\frac{1}{d(w)}\right)-\frac{1}{\eta^{2}} \\
& \quad \geq \frac{2}{n-1} \cdot\left(\frac{1}{d(v)}+\frac{1}{n-1}\right)-\frac{1}{n-1} \cdot\left(\frac{1}{d(v)}+\frac{1}{n-1-d(v)}\right) \\
& \quad=\frac{1}{n-1} \cdot\left(\frac{1}{d(v)}+\frac{2}{n-1}-\frac{1}{n-1-d(v)}\right) \\
& =\frac{1}{n-1} \cdot\left(\frac{(n-1)(n-1-d(v))+2 d(v)(n-1-d(v))-d(v)(n-1)}{d(v)(n-1)(n-1-d(v))}\right) \\
& =\frac{(n-1)^{2}-d(v)(n-1)+2 d(v)(n-1)-2 d(v)^{2}-d(v)(n-1)}{d(v)(n-1)^{2}(n-1-d(v))} \\
& =\frac{(n-1)^{2}-2 d(v)^{2}}{d(v)(n-1)^{2}(n-1-d(v))} .
\end{aligned}
$$

Therefore, the inequality that we want to prove becomes

$$
\begin{aligned}
& \frac{(n-1)^{2}-2 d(v)^{2}}{d(v)(n-1)^{2}(n-1-d(v))} \geq \frac{1}{(n-1) \eta} \\
& \Longleftrightarrow \\
& \frac{(n-1)^{2}-2 d(v)^{2}}{d(v)(n-1)(n-1-d(v))} \geq \frac{1}{\eta}=\frac{1}{\sqrt{d(v)(n-1-d(v))}} \\
& \Longleftrightarrow \\
& (n-1)^{2}-2 d(v)^{2} \geq(n-1) \sqrt{(n-1-d(v)) \cdot d(v)} .
\end{aligned}
$$

Now, since we are assuming $d(v) \leq \frac{n-1}{2}$,

$$
(n-1)^{2}-2 d(v)^{2} \geq \frac{(n-1)^{2}}{2} .
$$


Also, by applying $\sqrt{a b} \leq \frac{a+b}{2}$,

$$
(n-1) \sqrt{(n-1-d(v)) \cdot d(v)} \leq(n-1) \frac{n-1}{2}=\frac{(n-1)^{2}}{2} .
$$

Therefore,

$$
(n-1)^{2}-2 d(v)^{2} \geq(n-1) \sqrt{(n-1-d(v)) \cdot d(v)} .
$$

Thus, the proof of the lemma is complete.

Remark 4.3 In the particular case of $d_{\min }=\frac{n-1}{2}$, Theorem 4.1 tells us that

$$
\lambda_{n} \geq \frac{n+1}{n-1} .
$$

By the second part of Theorem 3.1, we know that this inequality is sharp.

Acknowledgements Open access funding provided by Project DEAL. The authors would like to thank the anonymous reviewer for the constructive comments.

Open Access This article is licensed under a Creative Commons Attribution 4.0 International License, which permits use, sharing, adaptation, distribution and reproduction in any medium or format, as long as you give appropriate credit to the original author(s) and the source, provide a link to the Creative Commons licence, and indicate if changes were made. The images or other third party material in this article are included in the article's Creative Commons licence, unless indicated otherwise in a credit line to the material. If material is not included in the article's Creative Commons licence and your intended use is not permitted by statutory regulation or exceeds the permitted use, you will need to obtain permission directly from the copyright holder. To view a copy of this licence, visit http://creativecommons.org/licenses/by/4.0/.

\section{References}

1. Atay, F.M., Tuncel, H.: On the spectrum of the normalized Laplacian for signed graphs: interlacing, contraction, and replication. Linear Algebra Appl. 442(1), 165-177 (2014)

2. Chung, F.: Spectral Graph Theory. American Mathematical Society, Providence (1997)

3. Das, K., Sun, S.: Extremal graph on normalized Laplacian spectral radius and energy. Electron. J. Linear Algebra 29(1), 237-253 (2016)

4. Li, J., Guo, J.-M., Shiu, W.C.: Bounds on normalized Laplacian eigenvalues of graphs. J. Inequal. Appl. 2014(1), 316 (2014)

5. Lin, H., Zhai, M., Gong, S.: On graphs with at least three distance eigenvalues less than -1 . Linear Algebra Appl. 458, 548-558 (2014) 